future studies could seek to validate the role of upper GI endoscopy in these referrals.

\begin{tabular}{lll}
\multicolumn{2}{c}{ Abstract PTU-099 Table 1: } & Cancers detected \\
\hline & Low Ferritin & \multicolumn{1}{c}{$\begin{array}{c}\text { Normal } \\
\text { Ferritin }\end{array}$} \\
\hline Colon & 14 & 3 \\
\hline Lung & 1 & 3 \\
Renal & 1 & 2 \\
Prostate & 1 & 1 \\
Others & Pancreas (1) & Stomach (2) \\
& Breast (1) & Oesophagus (1) \\
& Adrenal (1) & GOJ (1) \\
& & Lymphoma (1) \\
& & No primary (1) \\
\hline
\end{tabular}

\section{PTU-100 THE ROLE OF A MULTI-REGIONAL SPECIALIST MULTI- DISCIPLINARY MEETING IN DIAGNOSIS AND MANAGEMENT OF IGG4-RELATED DISEASE}

\begin{abstract}
1,2George Goodchild, ${ }^{3,4,2}$ Rory Peters, ${ }^{3,4}$ Tamsin Cargill, ${ }^{4}$ Adetokunbo Fadipe, ${ }^{1}$ Maria Leando, ${ }^{4}$ Raashid Luqmani, ${ }^{4}$ Ross Sadler, ${ }^{4}$ Eve Fryer, ${ }^{1}$ Louisa Firmin, ${ }^{1}$ Manuel Rodriguez-Justo, ${ }^{4}$ Helen Bungay, ${ }^{3,4,5}$ Eleanor Barnes, ${ }^{1,5}$ George Webster, ${ }^{3,4,5}$ Emma Culver. ${ }^{1}$ University College London Hospitals, London, UK; ${ }^{2}$ Contributed equally to the work; ${ }^{3}$ Translational Gastroenterology Unit, Oxford University, Oxford, UK; ${ }^{4}$ Oxford University Hospitals, Oxford, UK; ${ }^{5}$ Contributed equally to the work
\end{abstract}

\subsection{6/gutjnl-2019-BSGAbstracts.459}

Introduction IgG4-related disease (IgG4-RD) is a complex multi-system fibroinflammatory disorder, often presenting to the gastroenterologist as autoimmune pancreatitis (AIP) and sclerosing cholangitis (IgG4-SC). It requires careful diagnostic differentiation from malignancy and other inflammatory disorders. The majority of patients have persistent inflammation despite best available treatments, and many who achieve clinical remission subsequently relapse, leading to organ dysfunction and failure. Diagnostic challenges arise from multi-organ cross-speciality presentation, absence of a single diagnostic test and a plethora of diagnostic guidelines, whilst management decisions are influenced by the older demographic and identification of subclinical disease.

Methods We established an inter-regional IgG4-RD specialist multi-disciplinary meeting (MDM) held six weekly via weblink between Oxford and UCLH, incorporating multiple specialists across medical, surgical, immunology, histopathology and radiology disciplines. We describe our first year experience (Nov 2016-Nov 2017).

Results Over one-year, 50 patients were referred to the IgG4RD MDM. Of these 31/50 (62\%) had multiple organ involvement and $32 / 50(64 \%)$ had an elevated serum IgG4 $(>1 x U L N) .36(72 \%)$ were referred for diagnostic clarification, of whom 16 (44\%) met diagnostic criteria for IgG4-RD (Boston and/or CDC) or AIP/IgG4-SC (HISORt), 9 (25\%) did not meet diagnostic criteria but were considered to have 'possible' IgG4-RD and were managed as such, and 11 (31\%) did not meet diagnostic criteria, and an alternative diagnosis was offered in 6/11. Of those diagnosed with IgG4-RD, 25 (64\%) had management changes recormmended via the MDM, including treatment escalation $(21 ; 3$ of these had rituximab), de-escalation (4), conservative approach (14), further imaging and histology investigation (9), additional specialist opinion (3).

Conclusions We have demonstrated the value of a specialist IgG4-RD MDM incorporating a range of divesre specialists to aid diagnostic and management decsicions in a complex multisystem fibroinflammatory disease, providing a model for other centres across the UK.

\section{PTU-101 TRANSNASAL ENDOSCOPY; EARLY EXPERIENCE IN A SCOTTISH DISTRICT GENERAL HOSPITAL}

Gillian McColl, Elaine Yeap, Lynn Stirling, Rhona Inglis, Catherine Sharp, Kevin Robertson* University Hospital Crosshouse, NHS Ayrshire and Arran, Glasgow, UK

\subsection{6/gutjnl-2019-BSGAbstracts.460}

Introduction Per oral endoscopy can be uncomfortable and distressing leading many patients to opt for conscious sedation over topical local anaesthetic. Transnasal endoscopy is better tolerated with lower cardiovascular stress response. We sought to assess how easily TNE might be introduced to a District General Hospital and how acceptable patients found it.

Methods Patients requiring endoscopy were considered for TNE using $5.8 \mathrm{~mm}$ diameter Fujinon EG580NW2 scopes using topical nasal anaesthetic. Those with significant comorbidities, increased risk of bleeding and likely to need therapeutic intervention were excluded. Two consultants, with considerable OGD but minimal TNE experience, scoped consecutive patients from the outset of TNE introduction locally. Patients were sent postal feedback questionnaires including visual analogue scales (VAS 1 to 10) to assess comfort, distress, recollection of the peri-procedural consultation and overall experience.

Results 213 TNE procedures were performed by the consultants $(87 \%)$ and two senior trainees. For 6 patients $(2.8 \%)$ the scope could not be navigated through the nasal passages and endoscopy was completed, using the same scope, per oral. Two patients $(0.9 \%)$ had self-limiting epistaxsis and no patient required admission. 100 questionnaires (46.9\%) were returned. $61.1 \%$ of patients found their TNE procedure comfortable (VAS >6) with 17.9\% describing discomfort (VAS <5) $(21.0 \%$ ambivalent, VAS -). $72.9 \%$ did not find it a distressing procedure (VAS <5) whilst only $17.8 \%$ did (VAS $>6$ ), (9.4\% ambivalent, VAS -). $88.4 \%$ had a clear recollection of their consultation (VAS $>6$ ) with only 9\% reporting poor recollection (VAS <5), (5\% ambivalent, VAS -). Overall satisfaction was reported as good (VAS >6) by $94.7 \%$ of patients and poor (VAS <5) by only 5.3\%. Of our 213 patients, 33 had previous OGD experience with 28 (84.8\%) expressing preference for TNE. Our endoscopy database held reports for 18 of these showing 11 had Xylocaine spray and 7 intravenous Midazolam. Of the four patients that preferred OGD, 2 had had Xylocine and 2 Midazolam.

Conclusions TNE can be adopted by clinicians competent with conventional OGD with expectation of high procedure completion rate and low complication rate. Our findings suggest that our patients prefer TNE to conventional OGD, with or without sedation. Most patients found TNE comfortable with few reporting distress, maybe as a consequence, most patients had a clear recollection of their consultation. 